\title{
Desempenho de idosos em testes específicos: efeito de Grupo de Estimulação
}

Performance of healthy elderly in specific tests: effects of Cognitive Stimulation

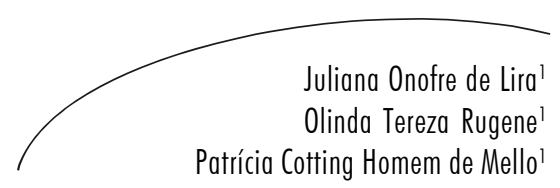

Resumo

Introducão: Com o envelhecimento, pode ocorrer um declínio cognitivo. Sabe-se que melhorar o desempenho das funções cognitivas em idosos tem consequências importantes em sua qualidade de vida. A literatura sugere que o treinamento pode aprimorar as funções cognitivas em idosos saudáveis. Objetivo: Verificar o efeito de grupo de estimulação cognitiva em idosos saudáveis. Métodos: Estudo longitudinal no qual foram avaliados 32 participantes escolarizados, com idade acima de 60 anos, divididos em dois grupos. O grupo-caso participou de 10 sessões de estimulação cognitiva em grupo. O grupo-controle foi formado por 15 indivíduos, com idade e escolaridade similares. A amostra foi avaliada antes e após a intervenção, através de uma versão adaptada da bateria CERAD, teste do relógio e check-list de queixas cognitivas. Foi usada uma análise descritiva, bem como o teste t-pareado, com o intuito de observar as diferenças entre os grupos após a estimulação. Resultados: Não houve diferenças entre os grupos quanto à idade, gênero e escolaridade. $\mathrm{Na}$ avaliação inicial, os grupos não apresentaram diferenças no desempenho dos testes aplicados. Na testagem após a intervenção, foi observada melhora apenas no grupo-caso nas tarefas de nomeação, fluência semântica, fluência total (bateria CERAD) e desenho do relógio, assim como na check-list de queixas cognitivas. Conclusão: A estimulação cognitiva realizada diminuiu as queixas cognitivas dos idosos participantes e melhorou seu desempenho em testes cognitivos.

\section{Abstract}

Background: Aging may be accompanied by cognitive decline. Improving cognitive function in elderly adults has important consequences in their quality of life. Literature suggests that training may improve cognitive functions in healthy elderly. Objective: To verify effect of cognitive stimulation group in healthy older adults. Methods: Longitudinal study in which we evaluated 32 educated participants above of 60 years, divided in 2 groups. The case group has participated

Palavras-chave: Cognição. Idoso. Estudos de intervenção. Serviços de saúde para idosos. Saúde do Idoso.

\footnotetext{
1 Instituto Paulista de Geriatria e Gerontologia “José Ermírio de Moraes”. Coordenadoria de Serviços de Saúde da Secretaria de Estado da Saúde. São Paulo, SP, Brasil. 
of 10 sessions of cognitive stimulation in group. And the other group was formed by 15 controls, with similar age and education. The sample was evaluated before and after intervention, using as instruments an adapted version of CERAD Neuropsychology Assessment, clock drawing test and check-list of cognitive complains. It was used descriptive analysis and Paired Student's t-Test to observe the differences between the groups after cognitive stimulation. Results: There were no differences between the groups for age, gender and education. In the initial evaluation, the groups did not present differences in the performance of applied tests. In the evaluation after intervention with cognitive stimulation, there was improvement only in the case group in the tasks of naming, semantic fluency, total fluency (CERAD battery) and clock drawing, as well as in the check-list of cognitive complaints. Conclusion: Cognitive stimulation applied has decreased the cognitive complains and has improved performance in cognitive tests in healthy older adults.
Key words: Self Stimulation. Cognition. Intervention Studies. Health Services for the Aged.

\section{INTRODUÇÃOO}

Vários estudos mostram que idosos apresentam muito comumente queixas relacionadas a funções cognitivas, principalmente a memória. ${ }^{1,2}$ Segundo Almeida, ${ }^{3}$ há estudos epidemiológicos que apontam que $5-54 \%$ dos idosos apresentam queixas relacionadas à memória. Um estudo no município de São Paulo constatou que $54 \%$ de pessoas acima de 65 anos de idade se queixam de sua memória, mas apenas $12 \%$ observam prejuízo nas atividades cotidianas pelas dificuldades com a memória. ${ }^{4}$ Se por um lado a queixa em si não é preditiva para o diagnóstico de demência, por outro ela parece correlacionar-se a sintomas depressivos e ansiosos. ${ }^{5,6}$ Tem-se encontrado maior frequência de queixa de memória entre as mulheres, o que pode relacionar-se com alteração hormonal pósmenopausa. ${ }^{3}$

As queixas de memória relacionam-se com a metamemória, conceito que se refere ao conhecimento objetivo de um indivíduo sobre a própria memória, quais tarefas são mais difíceis e quais as melhores estratégias para realizá-la e com a noção de autoeficácia. A autoeficácia, de modo mais amplo, é um aspecto da metamemória que diz respeito ao grau de certeza do indivíduo sobre sua capacidade de realizar uma tarefa envolvendo memória.?

As queixas cognitivas em idosos parecem estar. em muitos casos. mais relacionadas à percepção subjetiva negativa que o indivíduo tem do que com dificuldade objetiva no desempenho funcional. As queixas parecem, então, construirse em torno das crenças em relação ao envelhecimento. As crenças sobre a memória ou a noção de autoeficácia sobre a memória vêm sendo identificadas como um aspecto de grande influência sobre a cognição. ${ }^{8}$

Em outras palavras, o fato de o idoso apresentar queixas cognitivas não necessariamente implica que haja um déficit, mas pode-se tratar de uma supervalorização daquilo que esquece. ${ }^{3}$ Por outro lado, a significância clínica desta queixa é um tópico controverso, ${ }^{1}$ pois sabe-se que pode ocorrer uma queda no desempenho cognitivo com o envelhecimento típico e esta muito frequentemente é difícil de detectar em testes cognitivos, pois se caracteriza de forma muito específica. ${ }^{5}$ De qualquer forma, mesmo que a queixa seja decorrente de declínio cognitivo natural não relacionado a doenças, é importante a realização de intervenções que busquem melhorar o desempenho funcional do idoso, visto que este aspecto está relacionado à sua saúde, autonomia e qualidade de vida.,

Podemos verificar na literatura que há possibilidade de compensação da perda cognitiva em idosos saudáveis. Existem basicamente duas categorias na intervenção tradicional em cognição para idosos: treinamento de uma única técnica ao longo de uma ou duas sessões ou então o que 
envolve múltiplos procedimentos, entre 4 a 15 horas de duração. ${ }^{10}$ Nos dois tipos de intervenção, normalmente um instrutor treinado conduz esses programas e ensina(m) estratégia(s), modelos de como usá-la nas sessões, e também proporciona oportunidades para a prática na rotina com o feedback corretivo.

Alguns estudos têm avaliado a eficácia de diferentes intervenções cognitivas no envelhecimento típico. Rebok \& Balcerak ${ }^{11}$ verificaram o efeito de treino de memória em 48 adultos e 45 idosos através do método de loci e compararam com grupo-controle. Eles encontraram melhor desempenho nos jovens adultos, mas ambos os grupos apresentaram melhora nos testes utilizados após o treino.

Floyd \& Scogin ${ }^{12}$ realizaram uma meta-análise para verificar o efeito de treino de memória em indivíduos com queixa subjetiva e verificaram que a magnitude das queixas foi menor do que a observada através de testes específicos, mas não foram observados os desempenhos pré e pós-treino.

Ball et al. ${ }^{13}$ realizaram um estudo randomizado, duplo-cego, para avaliar a eficácia das intervenções através de exercícios para habilidades especificas da cognição - memória, velocidade de processamento e solução de problemas. Foram selecionados 2.832 idosos saudáveis voluntários divididos aleatoriamente em quatro grupos de intervenção, com dez sessões, grupo de treino de memória, grupo para solução de problemas, grupo para velocidade de processamento e grupo-controle, sem estimulação. Os idosos foram avaliados antes e imediatamente após a intervenção e em seguimento de dois anos. Verificou-se que houve melhora das habilidades específicas ( $87 \%$ velocidade de processamento; $74 \%$ solução de problemas; $26 \%$ treino de memória) após a intervenção. Os grupos-caso também apresentaram melhor desempenho durante o período do estudo nas atividades básicas e instrumentais de vida diária do que o grupo-controle.

Yassuda et al. ${ }^{8}$ estudaram 69 idosos saudáveis subdivididos em grupo experimental e grupo- controle. Após o término, os idosos do primeiro grupo, que foram submetidos à estimulação, fizeram uso mais intenso de estratégias de memória, no entanto foi verificado que mesmo assim não houve melhor desempenho quando comparado ao grupo-controle. Assim, os idosos treinados usaram a estratégia ensinada, mas não se beneficiaram dela.

Craik et al., ${ }^{2}$ após treino cognitivo, verificaram que idosos com queixas de memória, apresentaram melhor desempenho em testes em relação à memória secundária e processamento estratégico (resolução de problemas).

Apesar das queixas apresentadas serem principalmente de memória, todas as funções cognitivas são inter-relacionadas de forma que o desempenho em testes cognitivos complexos requerem a integridade de outras funções além da memória, que se constitui como foco específico da avaliação. Torna-se fundamental, portanto, uma mensuração adequada dos diversos aspectos cognitivos em idosos.

A partir deste dado, da relevância do tema na atualidade e das informações supracitadas, que mostram resultados não concordantes quanto ao benefício de estimulação cognitiva em idosos saudáveis, apresentamos o presente estudo, que foi realizado com o objetivo de avaliar a eficácia de intervenção em grupo de estimulação cognitiva em idosos participantes.

\section{METODOLOGIA}

Trata-se de estudo de intervenção desenvolvido no Centro de Referência do Idoso José Ermírio de Moraes (CRI), atual Instituto Paulista de Geriatria e Gerontologia (IPGG), em São Paulo.

A amostra foi composta por idosos inscritos para participar de grupo de estimulação cognitiva. Esse grupo ocorre na rotina de atendimento e é composto por uma demanda espontânea, bem como por encaminhamentos de profissionais do CRI de idosos saudáveis de ambos os gêneros que apresentam queixa cognitiva, sobretudo 
relacionada à memória. Os idosos participantes faziam acompanhamento clínico e não apresentavam suspeita diagnóstica de demência ou quaisquer alterações neurológicas. $\mathrm{E}$ antes de ingressar no grupo, o indivíduo foi avaliado para verificar seu desempenho em testes específicos. Somente foram incluídos no grupo os indivíduos com desempenho adequado no Mini-exame do Estado Mental (MEM) com escores estabelecidos por Bertolucci et al., ${ }^{14}$ na escala de Depressão Geriátrica - GDS ${ }^{15}$ e no Questionário de Pfeffer, ${ }^{16}$ respondido por um familiar próximo. Caso a performance fosse muito inferior aos escores estabelecidos pela literatura, o indivíduo era encaminhado ao serviço médico e não incluído no grupo. Após a existência de 20 idosos com os critérios para participação, foi iniciado o grupo. Este número foi escolhido devido ao espaço físico e critérios para formato de grupos educativos no serviço.

Para a realização deste estudo, foram avaliados 40 indivíduos, que foram divididos aleatoriamente em dois grupos:

- Grupo-caso: 20 idosos foram submetidos ao grupo de estimulação cognitiva. Este foi iniciado, no máximo, após duas semanas da realização da avaliação inicial. Com o término do grupo, estes idosos foram submetidos a uma reavaliação, com os mesmos instrumentos realizados na avaliação inicial.

- Grupo-controle: os 20 idosos restantes foram submetidos à avaliação inicial e, aproximadamente 12 semanas após esta, foram retestados. Após a reavaliação, eles foram convidados a participar do grupo de estimulação cognitiva.

Para a comparação do desempenho dos sujeitos, foram aplicados os seguintes testes na avaliação inicial e na reavaliação: para os aspectos cognitivos, foram aplicados o teste do relógio ${ }^{17} \mathrm{e}$ uma adaptação da bateria CERAD ${ }^{18}$ composta pela tarefa de memória de lista de dez palavras em três evocações imediatas (lista de palavras 1,2 e 3) e uma evocação tardia, bem como erros no reconhecimento destas, teste de nomeação de
Boston (Boston) em versão resumida com 20 figuras e fluência verbal semântica (animais) e fonológica (FAS) em um minuto; para mensurar as queixas de memória, foi aplicada uma check-list de itens de memória. ${ }^{19}$

\section{Grupo de estimulação cognitiva}

Foram realizados dez encontros semanais com duração de duas horas cada, sob coordenação de três profissionais e mais dois assistentes para auxiliar na realização dos exercícios. Os temas em cada seção: foram abordados temas com metodologia de fácil entendimento.

Nos dois primeiros encontros, além da apresentação e integração dos participantes, foram abordados de maneira facilitada os conceitos de cognição, suas funções e fisiologia. Desde o primeiro encontro, os idosos já foram expostos a exercícios para treino das funções cognitivas com ênfase em memória explícita, atenção e linguagem.

Na terceira sessão, foi abordado o tema "Mitos e verdades sobre a mente”, para discutir com o grupo sobre suas crenças referentes a cognição e sua relação com o envelhecimento. Foram discutidos e refletidos estes aspectos, aproximandose o saber dos profissionais à vivência cotidiana dos idosos. O principal objetivo desta sessão foi reduzir a ansiedade dos idosos quanto aos esquecimentos, debater a relação entre cognição e envelhecimento e melhorar a noção de autoeficácia, pois Yassuda $a^{20}$ observou um efeito positivo da ação educativa para modificar crenças negativas.

No quarto encontro, foi discutido o tema "Estilo de vida e sua influência no desempenho cognitivo". Através de tarefas solicitadas aos idosos, foi listado e discutido, posteriormente, o quanto hábitos diários podem interferir na saúde física e mental e, consequentemente, podem prejudicar ou ajudar o bom desempenho funcional. Foi discutida também a importância de se buscar alternativas para a mudança dos hábitos errôneos e da rotina diária.

O quinto encontro foi intitulado "Emoção: como nosso estado emocional pode afetar nossa 
memória? Resgate de lembranças significativas". Houve exposição do tema, e concomitantemente, discussão com os idosos, sobre como os aspectos emocionais e doenças como depressão e ansiedade podem interferir ou estarem associadas às dificuldades no dia a dia. Foi visto também como estresse, tensão e excesso de preocupações podem estar relacionados com a queixa cognitiva. Ao final, foram utilizados recursos visuais e auditivos como dispositivos para se recordar e falar sobre lembranças significativas e motivações para se lembrar ou esquecer delas.

$\mathrm{Na}$ metodologia utilizada, as estratégias para facilitar as atividades de vida diária foram divididas em internas, ou aquelas as quais necessitam de recursos cognitivos e externas, em que são necessários recursos ambientais. ${ }^{21}$

No sexto e sétimo encontros, foram abordadas as estratégias internas de categorização, associação, criação de imagem mental e organização. . $^{21,22}$

Nas sessões sobre estratégias externas $\left(8 .^{\mathrm{a}} \mathrm{e} 9 .^{\mathrm{a}}\right)$, foram apresentados ao grupo os dispositivos auxiliares de memória (agenda, calendário, blocos de anotações, recipientes dispensadores de medicação com alarme, mural de recados, lista de supermercados, organizadores de documentos). Em primeiro lugar, procurou-se estabelecer relação entre as estratégias externas já utilizadas no cotidiano e as apresentadas pelos terapeutas. Através da exposição dialogada, foram listados os dispositivos já utilizados e apresentados aqueles que não haviam sido citados. Essa apresentação contou com o suporte de recursos audiovisuais, expondo imagens dos dispositivos e comentandose sua forma de utilização. $\mathrm{Na}$ oitava sessão, recuperaram-se as informações obtidas na sessão anterior e abordou-se de forma prática o uso dos dispositivos auxiliares, solucionando-se dúvidas particulares, compartilhadas com o grupo. Treinou-se o preenchimento de uma agenda hipotética, procurando organizar os compromissos de acordo com a disponibilidade de tempo.

No décimo encontro, foram explanadas as relações entre as estratégias externas e internas.
Por exemplo, a utilização da categorização para a estruturação de lista de compras. Os objetivos dessa sessão foram introduzir e estimular o uso de dispositivos auxiliares de memória, de forma a diminuir a sobrecarga de informações cotidianas a serem armazenadas no cérebro e a evitar prejuízos para vida prática do idoso e para a saúde, como, por exemplo, pode ocorrer pelo esquecimento de uma consulta médica, ou da tomada da medicação.

Em todos os encontros foram aplicados diversos exercícios com o objetivo de realizar treino cognitivo, assim como foram passados exercícios relacionados para os idosos realizarem em casa.

A presente pesquisa foi aprovada pelo Comitê de Ética em Pesquisa do Instituto de Saúde da Secretaria da Saúde do Estado de São Paulo, sob o n. ${ }^{\circ}$ 0002/2009. A participação dos indivíduos no estudo foi condicionada a aceitação e assinatura de um termo de consentimento livre e esclarecido, que foi elaborado conforme recomendações do Conselho Nacional de Saúde, em respeito à Resolução no ${ }^{\circ} 196$, de 10 de outubro de 1996.

\section{RESULTADOS}

Foram recrutados 40 idosos para participar deste estudo: 20 para cada grupo. Cinco indivíduos que integravam o grupo-controle não compareceram à reavaliação e três não finalizaram o processo de intervenção. Desta maneira, a análise foi realizada com 17 indivíduos no grupo-caso e 15 no grupo-controle.

Não existiu diferença estatisticamente significante entre os grupos em relação aos anos de idade $(68,2 \pm 5,6$ versus $70,1 \pm 6,3$ anos; $95 \% \mathrm{IC}=$ $-6,1$ a 2,$4 ; \mathrm{t}(30)=-0,8 ; \mathrm{p}=0,393)$, escolaridade $(4,6 \pm 2,5$ versus $5,6 \pm 3,6$ anos; $95 \% \mathrm{IC}=-3,2$ a 1,2 ; $\mathrm{t}(30)=-0,9 ; \mathrm{p}=0,365)$, sexo $(70,6 \%$ versus $80,0 \%$ de mulheres; $\mathrm{p}=0,691)$, escores no GDS $(2,7 \pm 2,7$ versus $3,6 \pm 2,4 ; 95 \% \mathrm{IC}=-2,7$ a 0,$9 ; \mathrm{t}(30)=-0,9$; $\mathrm{P}=0,342)$ e MEM $(25,5 \pm 1,9$ versus $26,0 \pm 1,6$; $95 \% \mathrm{IC}=-1,7$ a 0,$8 ; \mathrm{t}(30)=-0,759 ; \mathrm{p}=0,454)$. 
Nas tabelas 1 e 2, são apresentadas as medidas descritivas em relação aos testes cognitivos aplicados na avaliação e reavaliação nos grupos caso e controle, respectivamente.

Tabela 1 - Análise descritiva do grupo-caso quanto aos testes cognitivos aplicados. São Paulo, SP, 2009.

\begin{tabular}{|c|c|c|c|c|c|c|}
\hline \multirow[b]{2}{*}{ Testes cognitivos } & \multicolumn{3}{|c|}{ Avaliação } & \multicolumn{3}{|c|}{ Reavaliação } \\
\hline & $\begin{array}{l}\text { Média } \\
( \pm \mathrm{DP})\end{array}$ & Mínima & Máxima & $\begin{array}{l}\text { Média } \\
( \pm \mathrm{DP})\end{array}$ & Mínima & Máxima \\
\hline Check-list & $10,4(3,8)$ & 5 & 15 & $6,4(4,2)$ & 1 & 15 \\
\hline Lista palavras 1 & $4,5(1,2)$ & 3 & 7 & $4,5(1,2)$ & 2 & 7 \\
\hline Lista palavras 2 & $6,0(1,3)$ & 4 & 9 & $6,7(1,4)$ & 4 & 9 \\
\hline Lista palavras 3 & $6,9(1,8)$ & 2 & 10 & $7,1(0,9)$ & 5 & 9 \\
\hline Evocação tardia & $5,3(1,3)$ & 3 & 8 & $6,1(1,5)$ & 3 & 9 \\
\hline Reconhecimento & $0,5(1,0)$ & 0 & 3 & $0,6(1,1)$ & 0 & 4 \\
\hline Boston & $15,8(3,9)$ & 5 & 20 & $17,1(2,9)$ & 9 & 20 \\
\hline Fluência Animais & $12,4(2,1)$ & 9 & 15 & $14,8(3,5)$ & 9 & 23 \\
\hline Fluência-F & $10,0(3,0)$ & 6 & 16 & $9,3(2,5)$ & 4 & 14 \\
\hline Fluência - A & $7,6(2,5)$ & 2 & 11 & $8,2(3,2)$ & 2 & 16 \\
\hline Fluência-S & $8,0(2,6)$ & 2 & 14 & $8,3(3,2)$ & 4 & 16 \\
\hline Total fluência & $38,1(8,0)$ & 20 & 53 & $40,7(9,3)$ & 22 & 55 \\
\hline Teste do relógio & $10,7(2,6)$ & 4 & 15 & $13,0(2,6)$ & 8 & 18 \\
\hline
\end{tabular}


Tabela 2 - Análise descritiva do grupo-controle quanto aos testes cognitivos aplicados. São Paulo, SP, 2009.

\begin{tabular}{lcccccc}
\hline \multirow{2}{*}{ Testes cognitivos } & \multicolumn{3}{c}{ Avaliação } & \multicolumn{3}{c}{ Reavaliação } \\
\cline { 2 - 7 } & Média & Mínima & Máxima & $\begin{array}{c}\text { Média } \\
( \pm \mathrm{DP})\end{array}$ & Mínima & Máxima \\
\hline Check-list & $10,7(4,3)$ & 4 & 20 & $9,8(5,5)$ & 2 & 20 \\
Lista palavras 1 & $4,7(1,2)$ & 3 & 7 & $5,2(1,3)$ & 3 & 8 \\
Lista palavras 2 & $6,6(1,4)$ & 5 & 10 & $7,0(1,6)$ & 4 & 10 \\
Lista palavras 3 & $7,0(1,4)$ & 4 & 10 & $7,8(1,2)$ & 6 & 10 \\
Evocação tardia & $5,8(2,1)$ & 0 & 8 & $5,9(3,0)$ & 0 & 10 \\
Reconhecimento & $1,0(1,7)$ & 0 & 5 & $1,0(1,6)$ & 0 & 5 \\
Boston & $16,4(3,3)$ & 9 & 20 & $16,6(3,5)$ & 8 & 20 \\
Fluência Animais & $13,3(3,9)$ & 8 & 19 & $13,8(3,8)$ & 7 & 20 \\
Fluência - F & $9,9(2,6)$ & 6 & 14 & $9,1(2,4)$ & 5 & 13 \\
Fluência - A & $7,2(3,0)$ & 1 & 11 & $8,4(3,0)$ & 2 & 12 \\
Fluência - S & $7,7(3,9)$ & 0 & 13 & $8,0(4,4)$ & 0 & 15 \\
To tal fluência & $38,6(10,4)$ & 19 & 51 & $39,8(10,9)$ & 18 & 57 \\
Teste do relógio & $10,9(4,2)$ & 3 & 15 & $11,6(4,2)$ & 4 & 15 \\
\hline & & & & & & \\
\hline
\end{tabular}

Foi aplicado o teste $t$ para verificar se os grupos mostravam diferenças em relação aos testes cognitivos aplicados antes da intervenção (tabela 3). 
Tabela 3 - Resultado da análise descritiva a partir das médias dos grupos quanto aos testes cognitivos aplicados na avaliação. São Paulo, SP, 2009.

\begin{tabular}{lcccccc}
\hline \multicolumn{1}{c}{ Testes cognitivos } & \multicolumn{2}{c}{ 95\% IC (diferença) } & $t$ & GL & $p$ \\
\hline Lista palavras 1 & & & & & 30 & 0,858 \\
Lista palavras 2 & $-1,0$ & A & 0,7 & $-0,327$ & 30 & 0,746 \\
Lista palavras 3 & $-1,5$ & A & 0,3 & $-1,244$ & 30 & 0,223 \\
Evocação tardia & $-1,2$ & A & 1,1 & $-0,099$ & 30 & 0,922 \\
Reconhecimento & $-1,7$ & A & 0,8 & $-0,712$ & 30 & 0,482 \\
Boston & $-1,5$ & A & 0,5 & $-0,932$ & 30 & 0,359 \\
Fluência Animais & $-3,2$ & A & 2,0 & $-0,446$ & 30 & 0,658 \\
Fluência - F & $-3,1$ & A & 1,3 & $-0,838$ & 30 & 0,409 \\
Fluência - A & $-1,9$ & A & 2,2 & 0,126 & 29 & 0,901 \\
Fluência - S & $-1,6$ & A & 2,4 & 0,361 & 29 & 0,721 \\
Total fluência & $-2,1$ & A & 2,7 & 0,355 & 29 & 0,813 \\
Teste do relógio & $-7,3$ & A & 6,2 & $-0,158$ & 29 & 0,875 \\
\hline
\end{tabular}

$\mathrm{Na}$ tabela 3, é possível verificar que não foram encontradas diferenças entre os grupos em nenhum dos testes aplicados na avaliação inicial. Desta forma, a seguir, através do teste t-pareado, analisou-se se houve diferença no desempenho dos indivíduos nos testes cognitivos após a intervenção do grupo de estimulação (tabela 4) 
Tabela 4 - Resultado da análise descritiva dos grupos no teste t-pareado quanto aos testes aplicados. São Paulo, SP, 2009.

\begin{tabular}{lcccccc}
\hline \multicolumn{1}{c}{ Testes cognitivos } & \multicolumn{3}{c}{ Grupo-caso } & \multicolumn{3}{c}{ Grupo-controle } \\
& Antes & Após & $p$ & Antes & Após & $p$ \\
\hline Check-list & 10,47 & 6,47 & $<0,001 *$ & 10,73 & 9,87 & 0,377 \\
Lista 1 & 4,59 & 4,53 & 0,835 & 4,73 & 5,20 & 0,250 \\
Lista 2 & 6,00 & 6,71 & 0,104 & 6,70 & 7,00 & 0,405 \\
Lista 3 & 6,94 & 7,12 & 0,713 & 7,00 & 7,80 & 0,054 \\
Lista tardia & 5,35 & 6,18 & 0,074 & 5,80 & 5,93 & 0,792 \\
Reconhecimento & 0,59 & 0,65 & 0,848 & 1,07 & 1,00 & 0,860 \\
Boston & 15,88 & 17,18 & $0,004^{*}$ & 16,47 & 16,67 & 0,792 \\
Animais & 12,41 & 14,82 & $0,011 *$ & 13,33 & 13,87 & 0,447 \\
F & 10,06 & 9,35 & 0,269 & 10,08 & 9,15 & 0,097 \\
A & 7,65 & 8,24 & 0,446 & 7,31 & 8,46 & 0,212 \\
S & 8,00 & 8,35 & 0,639 & 7,69 & 8,00 & 0,787 \\
Total fluência & 38,12 & 40,76 & $0,032^{*}$ & 38,69 & 39,85 & 0,493 \\
Teste do relógio & 10,76 & 13,06 & $0,002 *$ & 10,93 & 11,60 & 0,313 \\
\hline
\end{tabular}

* Diferença estatisticamente significante $(\mathrm{p}<0,05)$ pelo teste-t pareado.

Houve diferença estatística apenas no desempenho do grupo-caso, nos seguintes testes: check-list de queixas de memória, teste de nomeação de Boston reduzido, fluência verbal semântica categoria animais, total da fluência verbal e teste do relógio (tabela 4).

\section{DISCUSSÃO}

Foi observada melhora no desempenho de idosos saudáveis apenas nos participantes deste grupo de estimulação e isto ocorreu em alguns testes específicos aplicados: check-list de memória, teste de nomeação de Boston, fluência verbal e teste do relógio. $\mathrm{O}$ resultado nos demais testes não apresentou modificações significativas. É importante destacar que em nenhum momento da intervenção foram treinados os estímulos utilizados nos procedimentos de avaliação. Vários estudos também encontraram efeitos positivos de intervenções cognitivas em idosos. ${ }^{2,11,13,23,24}$

A metodologia utilizada nesta pesquisa englobou múltiplas estratégias de intervenção no formato de um grupo. Os resultados positivos encontrados sugerem que as intervenções com abordagens mais amplas podem produzir efeitos benéficos.

Foi possível verificar, através da check-list, que houve redução nas queixas em relação a aspectos cognitivos no grupo submetido à estimulação. Este dado pode ser justificado pelo fato de, nos encontros realizados, ter sido trabalhado o componente educativo para esclarecer crenças existentes a cognição e envelhecimento, assim como a prática da autopercepção do desempenho funcional na rotina diária. Com os resultados obtidos, foi verificado que essa abordagem se mostrou eficaz. 
Yassuda ${ }^{8}$ ressalta que os estudos sobre a relação entre queixas (autoeficácia) e sucesso no treino cognitivo precisam ser aprimorados, mas possivelmente a melhora na noção de autoeficácia pode ser um fator relevante para o sucesso do treino.

Em ambos os grupos, houve na avaliação inicial uma alta frequência de queixas, visto que a amostra foi composta por indivíduos que procuraram espontaneamente a intervenção. Todos os idosos foram investigados e não apresentavam doenças relacionadas à cognição. Isto reforça a ocorrência de uma supervalorização das queixas, assim como verificado por Almeida. ${ }^{3}$

Em relação ao teste de nomeação de Boston, observou-se melhor desempenho no grupo-caso após a intervenção. Tanto neste teste ou na fluência verbal, as funções cognitivas mais demandadas são memória semântica e linguagem, as quais sofrem um menor efeito da idade, ${ }^{25}$ provavelmente ocasionado pela larga experiência linguística ao longo da vida. Somando este dado ao nosso achado, podemos supor que há maior facilidade no treino de atividades que envolvem funções mais “intactas”. Isto pode ser considerado um mecanismo de proteção cognitiva relacionado a um desempenho funcional mais eficiente. ${ }^{26}$

O grupo-caso também apresentou melhora no desempenho no teste do relógio. Este teste avalia várias funções cognitivas, com destaque para as funções executivas, que são responsáveis pelo planejamento e execução de tarefas. ${ }^{27}$ No grupo de estimulação cognitiva, foram intensamente trabalhadas técnicas de organização e desenvolvimento de estratégias para desempenhar as atividades na rotina diária dos idosos de maneira eficaz. Assim, esta abordagem pode ter ocasionado a melhora no teste citado na reavaliação do grupo-caso.

Outros testes não apresentaram diferenças após a intervenção realizada. Estes são voltados mais à atenção, memória de trabalho e memória episódica. Segundo Taussik \& Wagner, ${ }^{28}$ há efeitos significantes do envelhecimento nas funções cognitivas supramencionadas. A intervenção realizada neste estudo apresentou duração de dez encontros, com abordagem de vários temas. Apesar de terem sido incluídos na amostra apenas idosos saudáveis, para compensar modificações provocadas pela idade talvez seja necessário um maior número de sessões.

Outro aspecto importante a ser abordado é a escolaridade. A maioria dos integrantes da pesquisa apresenta poucos anos de estudo. Segundo Argimon \& Stein, ${ }^{29}$ a alta escolaridade confere importante papel nas habilidades cognitivas dos idosos e pode facilitar o bom desempenho ligado a algumas funções. Na prática da intervenção feita no presente estudo, muitas vezes, os idosos tinham dificuldades em compreender rapidamente as instruções do treino e desta forma, para garantir a qualidade da resposta, houve uma lentificação do processo de explicação e execução das tarefas propostas. Este pode ter sido outro fator que influenciou o desempenho nos testes que se mantiveram com escores semelhantes na reavaliação. Esta análise reforça que talvez tenha que ocorrer um aumento no número de encontros ou mesmo diminuir a quantidade de indivíduos.

\section{CONCLUSÃO}

Os idosos participantes do grupo de estimulação apresentaram melhor desempenho na reavaliação em testes de memória semântica, linguagem, funções executivas, assim como diminuição das queixas relatadas.

Os achados deste estudo refletem a eficácia da intervenção realizada, considerando que foi realizada uma abordagem grupal e em apenas dez sessões. Desta forma, é suposto que uma intervenção com maior duração poderá proporcionar melhor desempenho nos demais testes aplicados.

Esta pesquisa foi realizada a partir da rotina de um ambulatório existente voltado à população idosa no nível secundário do atendimento do Serviço Único de Saúde (SUS). Assim, os resultados verificados são muito importantes para a modificação da prática profissional. 
Vale ressaltar que os procedimentos de avaliação realizados foram quantitativos, utilizando escalas e testes que sofrem influência da escolaridade e de aspectos socioculturais. Uma abordagem qualitativa pode ser mais abrangente no intuito de analisar o resultado subjetivo da intervenção.

\section{REFERÊNCIAS}

1. Mattos P, et al. Memory complaints and test performance in healthy elderly persons. Arq Neuro-Psiquiatr 2003;61(4):920-924.

2. Craik FIM, Winocur G, Palmer H, Binns MA, et al. Cognitive rehabilitation in the elderly: effects on memory. J Int Neuropsychol Soc 2007; 13: 132-42.

3. Almeida OP. Mini-exame do estado mental e o diagnóstico de demência no Brasil. Arq NeuroPsiquiatr 1998; 56(3B): 605-12.

4. Brucki SMD, et al. Consortium to establish a registry for Alzheimer's disease : aspectos epidemiológicos. Arq Neuro-Psiquiatria 1994; 52(suppl): 1-9.

5. Bertolucci PHF. Demências. In: Ortiz KZ. (Org.). Distúrbios neurológicos adquiridos. Barueri: Manole; 2005. p.295-312.

6. Guerreiro TC ,et al. Queixa de memória e disfunção objetiva de memória em idosos que ingressam na Oficina de Memória na UnATI/ UERJ. Rev. bras. geriatr gerontol 2006; 9(1): 7-21.

7. Yassuda MS, Lasca VB, Neri AL. Meta-Memória e Auto-eficácia: um estudo de validação de instrumentos de pesquisa sobre memória e envelhecimento. Psicologia: reflexão e crítica 2005; 18(1): 78-90.

8. Yassuda MS ,et al. Treino de memória no idoso saudável: benefícios e mecanismos. Psicologia: reflexão e crítica 2006; 19(3):470-81.

9. Ramos LR. Fatores determinantes do envelhecimento saudável em idosos residentes em centro urbano: projeto Epidoso, Cad Saúde Pública 2003; 19: 793-797.

10. Rebok GW, Carlson MC, Langbaum JC. Training and maintaining memory abilities in healthy older adults: traditional and novel approaches. Journals of gerontology 2007;62(1):53-61.

11. Rebok GW, Balcerak LJ. Memory self-efficacy and performance differences in young and old adults: the effect of mnemonic training. Developmental Psychology 1989; 25(5): 714-21.

12. Floyd M, Scogin F. Effects of memory training on the subjective memory functioning and mental health of older adults: a meta-analysis. Psychol Aging 1997;12(1):150-61.
13. Ball $\mathrm{K}$,et al. Effects of cognitive training interventions with older adults: a randomized controlled trial. Jama 2002; 288(18): 2271-81.

14. Bertolucci PHF ,et al. O mini-exame do estado mental em uma população geral: impacto da escolaridade. Arq Neuro-Psiquiatr 1994; 52(1): 1-7.

15. Yesavage JA, et al. Development and validation of a geriatric depression screening scale: a preliminary report. J Psychiatr Res 1983; 17: 3749.

16. Pfeffer RI et al. Measurement of functional activities in older adults in the community. J Gerontol 1987; 37: 323-9.

17. Freedman M, Leach L, Kaplan E, et al. Clockdrawing: a neuropsychological analysis. $\mathrm{New}$ York: Oxford University Press; 1994.

18. Bertolucci PHF, et al. Aplicabilidade da bateria neuropsicológica CERAD em idosos brasileiros. Arq Neuro-Psiquiatr 2001; 59(3A): 1-7.

19. Alvarez A, et al. Memória. São Paulo: Atheneu; 2005.79p.

20. Yassuda MS. Memory beliefs and memory training: the effects of an educational intervention. Gainesville .Tese. -University of Flórida; 1999.

21. Alvarez A. Deu branco: um guia para desenvolver o potencial de sua memória. São Paulo: Best Seller; 2002.

22. Gediman CL, Crinella FM. Deixe seu cérebro em forma. Rio de Janeiro: Sextante; 2005. 271p.

23. Newson R, Kemps E. The Influence of Physical and Cognitive Activities on Simple and Complex Cognitive Tasks in Older Adults. Aging clin expres 2006; 32(3): 341-362.

24. Scarmeas $\mathrm{N}$,et al. Influence of leisure activity on the incidence of Alzheimer's Disease. Neurology 2001; 57: 236-242.

25. Mitchell DB. How many memory systems? Evidence from aging. J exp. psychol: learning, memory and cognition 1989; 15:31-49.

26. Park, et al. Improving cognition function in older adults: nontraditional approaches. J gerontol 2007; 62(1):45-52. 
27. Ashman T, Mohs R, Harvey P. Cognition and aging. In: Hazzard WR ,et al. Principles of geriatric medicine and gerontology. USA: McGraw-Hill; 1999. p. 1219-1228.

28. Taussik I, Wagner GP. Memória explícita e envelhecimento. In: Parente MAMP (Org.).
Cognição e envelhecimento. Porto Alegre: Artmed; 2006. p. 67-84.

29. Argimon IIL, Stein LM. Habilidades cognitivas em indivíduos muito idosos: um estudo longitudinal. Cad. Saúde Pública 2005; 21(1): 6472.

Recebido: 04/1/2010

Revisado: 06/8/2010

Aprovado: 24/9/2010 